\title{
26474 - CORONARY SINUS LACTATE CONCENTRATION DURING MYOCARDIAL REPERFUSION
}

\author{
Ashraf Fayad FCARCSI, FACC, Homer Yang, ; Labib Abib, \\ University Of Ottawa, Ottawa, ONTARIO, Canada
}

Introduction:

Weaning from cardiopulmonary bypass (CPB) after open heart surgery is usually uncomplicated step. Occasionally the separation process could be very difficult and may require the use of pharmacological or mechanical ventricular supports. The recovery time for the myocardium is usually based on the clinical experience. The purpose of this study is to examine the coronary sinus lactate concentration during myocardial reperfusion in open-heart surgery.

Methods:

Patients undergoing elective aortic valve replacement, coronary artery bypass grafting (CABG) or both were enrolled in an observational study. Pre-operative cardiac function, lesions, and patient demographics were documented. Anesthetic and surgical techniques were as per institutional practice. Myocardial protection was provided by alternating antegrade and retrograde warm blood cardioplegia (4:1 dilution). Blood samples from the retrograde cannula were collected prior to $\mathrm{CPB}$, immediately after releasing the aortic cross clamp and every 3 minute thereafter until the separation time. Samples were analyzed using a Nova Plus L blood gas machine. The CPB time, aortic cross clamp, time to separate from CPB, number of attempts and the use of inotropes were also recorded. Results:

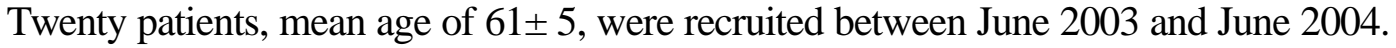
Nine patients underwent aortic valve replacement (AVR), three AVR + CABG and eight CABG. The cardioplegia arrest time was $133 \pm 41$ minutes (range, 64 to 275 minutes). The lactate levels are in Table 1 . Sixteen patients had lactate level of less than 4 meq/L immediately after aortic cross clamp: 15 patients were weaned off CPB on the first attempt without pharmacological support; one required inotropes and was weaned off $\mathrm{CPB}$ on the second attempt. The reperfusion time in this group ranged between $6-18$ minutes. The remaining 4 patients had lactate concentrations higher than 4 meq/L immediately after aortic cross clamp: all required a pharmacological support and more than one attempt to come off CBP. The average reperfusion time in this group was $18-$ 21 minutes. In all patients, the coronary lactate concentration didn't change significantly over the reperfusion time.

Discussion:

The reperfusion time is usually based on clinical experience1,2,3 and some surgeons may elect to "rest" the heart for long periods of time. The length of time to "rest" the heart varies and the optimal length of time has not been described. The coronary lactate concentrations in this study did not change significantly over reperfusion time, and thus, prolonged resting of the heart does not seem to confer additional advantages. However, patients with high lactate (more than 4) concentration during the reperfusion are more likely to require inotropes to wean from CPB. 
Coronary Sinus Lactate Concentration During Myocardial Reperfusion

\begin{tabular}{|c|c|c|c|c|c|}
\hline Immediately & $3 \min$ & $6 \mathrm{~min}$ & $9 \min$ & $12 \mathrm{~min}$ & $15 \min$ \\
\hline 1.8 & 1.6 & 1.5 & 1.4 & 1.4 & \\
\hline 3.1 & 3 & 2.9 & & & \\
\hline 5 & 4.6 & 4.2 & 4.3 & 4.7 & 4.9 \\
\hline 8.9 & 8.3 & 7.8 & 7.5 & 7.8 & 7.9 \\
\hline 2.3 & 2.2 & 2 & 2.3 & & \\
\hline 2.1 & 2 & 2 & & & \\
\hline 4.7 & 4.5 & 4.4 & 4.3 & 4.4 & 4.5 \\
\hline 2.2 & 2.1 & 2 & 1.9 & 1.8 & 1.9 \\
\hline 2.8 & 2.3 & 2.1 & 2.1 & 1.9 & 2.1 \\
\hline 6.5 & 4.5 & 4.1 & 3.9 & 3.6 & 3.4 \\
\hline 2.6 & 2.3 & 2.3 & 3.3 & & \\
\hline 3.7 & 2.7 & 2.4 & 2.7 & 2.7 & 2.9 \\
\hline 2.8 & 2.6 & 2.7 & 2.7 & 2.3 & 2.7 \\
\hline 2.2 & 2.2 & 2.2 & 2.2 & & \\
\hline 3.6 & 3.1 & 28 & & & \\
\hline 1.6 & 1.3 & 1.1 & 1.7 & & \\
\hline 3.2 & 3.3 & 3.9 & & & \\
\hline 2.1 & 2.1 & 1.9 & 1.8 & 1.9 & \\
\hline 3.7 & 3.6 & 3.6 & & & \\
\hline 1.6 & 1.5 & 1.5 & 1.8 & 1.8 & \\
\hline 3.3 & 3 & 2.9 & 3 & 3.3 & 3.6 \\
\hline SD1.809587042 & 1.586754384 & 1.508606886 & 1.579089651 & 1.91144876 & 1.970813828 \\
\hline
\end{tabular}

